\title{
IMMERSIVE TECHNOLOGIES IN 5G-ENABLED APPliCATIONS: SOME TECHNICAL CHALlENGES OF THE NOVEL USAGE MODELS
}

\author{
Nikola Rendevski, Andrijana Bocevska, Zoran Kotevski and Tome Dimovski \\ Faculty of Information and Communication Technologies, \\ University St. Kliment Ohridski - Bitola, Macedonia
}

\begin{abstract}
$5 G$ next-generation networking paradigm with its envisioned capacity, coverage, and data transfer rates provide a developmental field for novel applications scenarios. Virtual, Mixed, and Augmented Reality will play a key role as visualization, interaction, and information delivery platforms. The recent hardware and software developments in immersive technologies including $A R, V R$ and $M R$ in terms of the commercial availability of advanced headsets equipped with XR-accelerated processing units and Software Development Kits (SDKs) are significantly increasing the penetration of such devices for entertainment, corporate and industrial use. This trend creates next-generation usage models which rise serious technical challenges within all networking and software architecture levels to support the immersive digital transformation. The focus of this paper is to detect, discuss and propose system development approaches and architectures for successful integration of the immersive technologies in the future information and communication concepts like Tactile Internet and Internet of Skills.
\end{abstract}

\section{KEYWORDS}

5G, Virtual Reality, Augmented Reality, Immersive Technologies, Extended Reality, Information Systems.

\section{INTRODUCTION}

The implementation of the fifth-generation mobile network $5 \mathrm{G}$ is currently happening globally in both the developed and developing world. Besides the serious radio network infrastructure investments caused by the move from micro- to millimeter-wave bands [1], the mobile networks service providers have determined the future business potential and income generation models. Day-by-day the availability of certain 5G-envisioned characteristics such as guaranteed gigabit data transfers, improved coverage, and sub-millisecond latencies are becoming reality.

Several novel 5G-enabled technology concepts inspired by the 5G envisaged capabilities emerged in the past 7 years. In this paper we will consider two of the most promising technological visions, to analyse and detect the main technical challenges coming from the massive involvement of immersive technologies (Augmented Reality, Virtual Reality, and Mixed Reality) as one of the key elements for interaction and information delivery in their ecosystems: Tactile Internet [2,3] and Internet of Skills [4].

\section{VR, AR, MR AND XR}

The technologies we collectively refer to as XR (eXtendedReality) nowadays are Augmented Reality (AR), Virtual Reality (VR), and Mixed Reality (MR), which includes other hybrid, 
immersive experiences. Figure 1 depicts the XR technologies diagram showing the Mixed Reality (MR) as the space where AR and VR technologies overlap. Virtual Reality (VR) is a computergenerated artificial environment where the scenes and objects in the user visual feed are artificial (digital) making the user to feel as immersed in the virtual world. On the other side, Augmented Reality (AR) allows the user to see the real environment combined with digital objects overlaid in the physical space. Mixed Reality (MR) not only merges the physical and digital world, as it is purely AR characteristic but creates physical-digital coexistence and interaction known as a hybrid of reality. In the past decade, the advancements in both hardware and software development platforms in all three domains enabled the significantly easier creation of various XR applications applicable in various areas of industry, medicine, science, and entertainment.

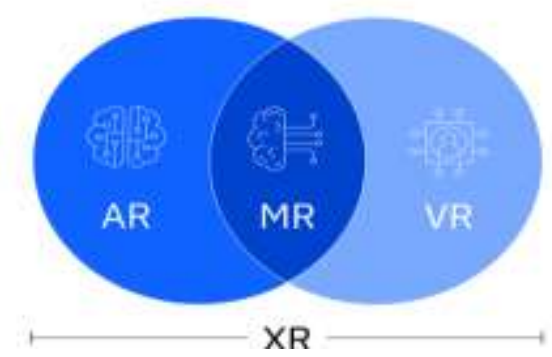

Figure 1. Extended Reality, Augmented Reality, Mixed Reality and Virtual Reality Diagram (source: IBM)

\section{IMMERSIVE TECHNOLOGIES IN THE TACTILE INTERNET CONCEPT}

The term Tactile Internet (TI) was coined in 2014 by Professor Gerhard Fettweis from the Technical University of Dresden, Germany. Later in the same year, International Telecommunication Union (ITU) defined it in its Technology Watch from the aspect of the existing standards and their potential impact on future standardization. Tactile Internet paradigm aims to contribute to the solution of the complex challenges faced by modern societies such as education and lifelong learning, healthcare, personal safety zones, smart city, energy. Tactile Internet is envisioned to create a paradigm shift from content-oriented communication to control-based communication by allowing real-time transfer of haptics like touch, motion, vibration, surface texture over the network along with the conventional audio and visual data. TI as the next evolution stage of the Internet of Things (IoT), is expected to create numerous business opportunities for applications in various technology markets.

The shift from content-oriented to control-oriented communication, from the other side, creates a guaranteed and important role for immersive technologies as Virtual Reality, Augmented Reality, and Mixed Reality. The general challenges from the aspect of technical requirements for networking architectures, processing models, software architectures, virtualization, and haptics within the TI are widely investigated in academia and industry.

Virtual Reality (VR) video streaming is considered the best-suited technology to provide the immersive visual feed towards achieving the envisioned levels of real-time interaction in TI. In [5] the authors pinpointed the open challenges to enable VR video streaming technologies for the Tactile Internet through analysis of the state-of-the-art on VR video streaming technologies both, theoretically and by means of an experimental demonstrator. It was shown that to achieve the real-timeliness (maximum 5 milliseconds end-to-end latency) and link quality (5 Gbps data transfer speed) required by the TI, VR content streaming needs to overcome several challenges such as improvements of transport and application protocols, improvements of the edge 
infrastructure to migrating the processing-intensive tasks from the processing constrained VR units and enabling precise synchronization of the complex haptics sensor systems within VR scenes. The VR technical challenges pointed in [5] obviously stand for Augmented and Mixed/Extended Reality applications also, as the currently available and future AR/MR headset devices and platforms practically share the processing units with VR in terms of chip architectures and processing power needed to render the digital content and process the sensor data feed to navigate in the virtual/mixed environment.

To analyze and discuss the VR / AR / MR technical challenges in the TI environment, within all hardware and software design architecture layers, we must precisely define the new elements that TI will originally incorporate in the VR technology which development was certainly independent of TI concept in the past decade. One of the main differences the of TI immersive environment from the classic XR applications is certainly the so-called Shared Haptic Virtual Environment (SHVE), where several users are physically coupled via XR simulation to perform tasks that require fine-motor skills. Haptic feedback is a prerequisite for a high level of interaction [6], allowing the user to perceive the objects in the XR not only by audio and visual feed but also via the sense of touch. Such an environment allows manipulating with objects as envisioned in remote medical interventions, complex robotized machinery remote operation, micro-assembling, and numerous other applications sharing common important technical demands such as high level of sensitivity, and precision. The SHVE's are creating a direct impact on the immersive applications from several aspects. First, from the hardware perspective, TI requires coupling and integration with complex input/output XR controllers with capabilities for haptic input and feedback. Second, from software and hardware aspects, the so-called multiplayer/multiuser VR environments should significantly evolve in terms of providing reliable and low latency communications in both haptic controller-XR local physical connections and XR to XR device distant communications. In the following, we will analyze and discuss both, haptic controller connections in the SHVE and the networking aspects towards achieving high-speed communication and sub-millisecond latencies.

\subsection{Haptic Rendering and SHVE}

Haptic rendering is the process of calculating and applying force feedback to the user's haptic device directly from the virtual environment. The implementation of the haptic rendering technique depends on the application requirements. In [7], the authors classified the SHVE architectures in three major classes: static, collaborative, and cooperative shared virtual simulations and analysed two different approaches of haptic rendering: impulsive haptic rendering and continuous haptic rendering. In a cooperative virtual environment, users can simultaneously modify and interact with the same virtual object. In this paper, we will focus on the continuous haptic rendering in cooperative SHVE since it corresponds to the abovementioned mission-critical Tactile Internet application scenarios.

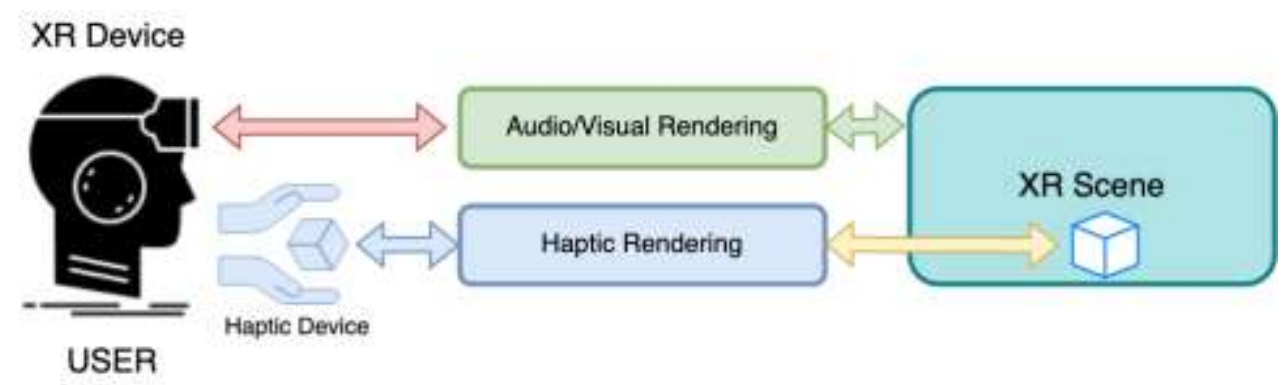

Figure 2. Basic Architecture of a XR application with haptic feedback 
Figure 2 illustrate basic interaction flow architecture of a XR application with haptic feedback. From the image, we can conclude that besides the classic audio-visual feed, in such applications haptic devices are present and the communication with the haptic devices is happening outside the XR feed physically, but the actions are or might be caused by interaction with a digital object in the XR scene. This digital object can be representation of a real object or virtual simulation of the real object behaviour.

In contrast to regular displays which are stimulating other human senses, the haptic devices create bidirectional energy flow, to and from the operator. This creates a feedback loop consisted of the user, XR device, haptic device, and haptic rendering mechanism. It is well known that such loops can create instability and the problem of stable haptic interaction in general has attracted serious attention in the past decade. In [8], two main sets of techniques were classified which can be adopted in the contemporary immersive environments:

- Using virtual damping to limit the flow of energy from virtual environment toward user when this could create unstable behaviour, and

- Decoupling force response algorithms from other slower algorithms, such as the ones computing collision detection, visual rendering, and virtual environment physics.

With the concept of virtual coupling, virtual environments can be created allowing unlimited feedback levels, but the haptic device will render only the maximum level set by the virtual coupling. Decoupling haptic response algorithms from other slower algorithms, provides a simpler local representation of a virtual object at the highest possible rate that can be achieved on the system. This can be realized by running all the algorithms in different threads featuring different haptic feedback rates.

Considering the above-mentioned facts and the characteristics of the Tactile Internet concept such as sub-millisecond latencies and the stability required for the envisioned mission-critical applications, it is obvious that nowadays there are no sophisticated immersive application development environments nor standards and protocols for interfacing the haptic devices in the complex next-generation immersive environments. In fact, these are the main technical challenges to be solved: the creation of Haptic Devices Pairing Protocols (HDPP) and API architectures forbi-directional logical communication of the XR scene and the haptic devices.

\subsection{Haptic I/O Controllers and XR Devices Coupling}

Most of the commercially available XR headsets nowadays don't share universal protocols for coupling with external devices. This is certainly possible, but it requires deep knowledge of the hardware platform (XR headset devices) and the platform-specific software development environment to enable input/output to/from the XR scene.

Since the haptic transfer, interaction, and feedback set is finite in terms of the physical interaction senses, such as force, motion, texture, touch, temperature, pressure, etc., it is naturally expected that with the evolution of such devices, the need for XR platform-independent integration will be necessary for rapid development of next-generation immersive applications and services.

It is well known that a human can distinguish various stimulations within three sensory systems:

- Cutaneous receptors (found in the skin) [9]. Cutaneous receptors include mechanoreceptors (pressure or distortion), nociceptors (pain), and thermo receptors (temperature).

- Kinaesthetic (body motion and perception of the motion) 
- Haptic (experiencing the surfaces of various objects and object recognition)

There are several ways to implement the senses in the tactile devices as vibration, force feedback, and temperature sensation.

Vibration is one of the most common ways that haptic devices use to generate haptic feedback. It can be implemented as ERM (Eccentric Rotating Mass) - rotating non-balanced weight [10], [11] and LRA (Linear Resonant Actuator)- converting electrical signals into motion.

Force feedback devices use electric motors to manipulate the movement of an item held by the user. Nowadays, there are numerous commercial solutions for providing force feedback - widely used in gaming controllers.

The thermal sensation [12] is a cutaneous sense also. The thermal haptic devices, in most cases, use IR (infrared radiation) to heat surfaces of interest where the thermal sensation should be provided.

Figure 3 presents a proposed architecture flow for physical coupling of the haptic devices and API block for enabling the logical high-level communication with the XR software application.

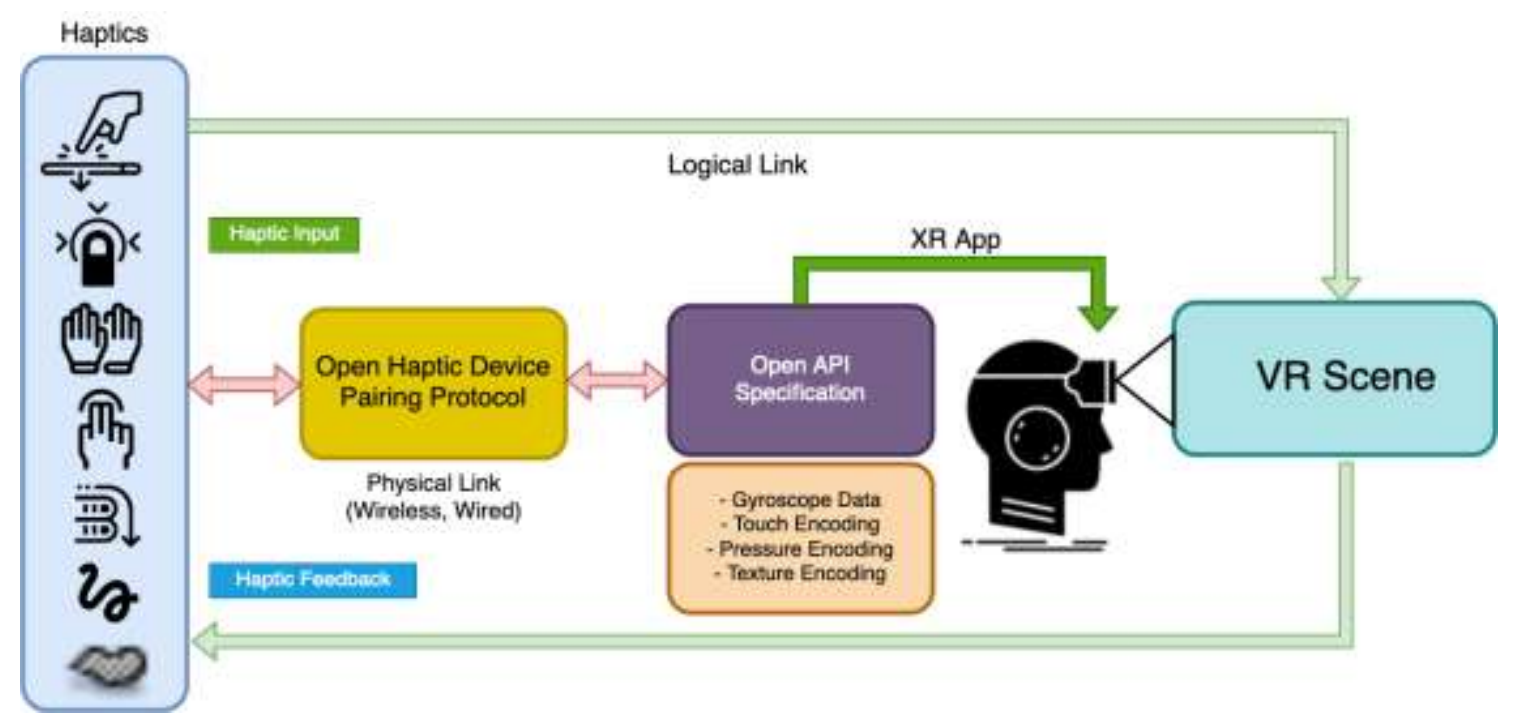

Figure 3. Proposed architecture for haptic devices coupling in XR

The open API block should contain the abstraction of all the functions and senses the device can deliver to and emulate from the XR scene. If we assume that all the input and feedback from and to the haptic device is falling within the main sense-providers such as vibration, force feedback, and temperature, then one may conclude that each feedback or input corresponding with the sense that has to be delivered is a combination of senses to the exact device. In other words, the device should receive/provide a set of instructions from/to the API, to encode the sense of interest such as texture, pressure (force), temperature, etc. The creation of highly abstracted open API specifications for logical communication with the XR devices (XR scenes) may provide a huge developmental field and stimulate the creation of various haptic devices capable to be used in the Tactile Internet XR scenarios. Figure 4, presents exemplary API abstraction parameters which provide: 
- Device ID (Selecting the device bydevice_id)

- Intensity (intensity of the feedback to be provided such force, vibration, temperature)

- Frequency (vibration, linear motion, rotation, etc.)

- Type (force, vibration, temperature)

- Subtype (method for providing the sense such ERM, LRA)

- Duration (time duration of the certain sense applied)

The existence of a universal Open API can guarantee that the creators of the next-generation haptic devices will be able to connect the devices with XR (AR/MR/VR) platforms and realize the logical communication with the virtual objects in the XR scenes. Regarding the physical network connection of the haptic device with the XR headset, various technologies may be used. Currently, Bluetooth connections are dominating but since the sensor fusion data may significantly increase, the low bandwidth communication technologies may be inefficient to provide the data rates required to transmit and receive massive sensor data. As an alternative, next-generation Wi-Fi technologies may be used or even $60 \mathrm{GHz}$ bands [13],[14] which allow huge data rates and low latency in the case of real-time multimedia content transmission.

\section{Conclusions}

In this paper, we focused to detect several challenges for successful implementation of the XR in the next-generation communication concepts as Tactile Internet. The paradigm shift from content-oriented communication to control-based communication requires real-time transfer of haptics over the communication links along with the conventional audio and visual data. Currently, the development of the XR technologies and the advancement of haptic devices are unrelated and such situations produce numerous technical difficulties for co-existence and integration. We emphasized the serious need for open API specifications (from a software design perspective) and the design of effective protocols for physical communication between the XR devices and the haptic controllers and proposed initial development architecture which will enable the integration of the SHVE in the next generation so-called metaverses. As the senses and methods for their realization are a finite well known set, the standardization in the haptic devices design, pairing and communication mechanisms, and software design is a real requirement for the advancement of the next-generation concepts such as Tactile Internet and Internet of Skills where the XR technologies will play a key role. As future work, we plan to further investigate the tactile haptic device architectures and propose a more precise definition of the communication protocols and universal API structure capable to provide a mechanism for efficient involvement of the future haptic devices in the XR technology platforms.

\section{REFERENCES}

[1] S. Painuly, S. Sharma and P. Matta, "Future Trends and Challenges in Next Generation Smart Application of 5G-IoT," 2021 5th International Conference on Computing Methodologies and Communication (ICCMC), 2021, pp. 354-357, doi: 10.1109/ICCMC51019.2021.9418471.

[2] M. Simsek, A. Aijaz, M. Dohler, J. Sachs and G. Fettweis, "5G-Enabled Tactile Internet," in IEEE Journal on Selected Areas in Communications, vol. 34, no. 3, pp. 460-473, March 2016, doi: 10.1109/JSAC.2016.2525398.

[3] S. K. Sharma, I. Woungang, A. Anpalagan and S. Chatzinotas, "Toward Tactile Internet in Beyond 5G Era: Recent Advances, Current Issues, and Future Directions," in IEEE Access, vol. 8, pp. 5694856991, 2020, doi: 10.1109/ACCESS.2020.2980369.

[4] M. Dohleret al., "Internet of skills, where robotics meets AI, 5G and the Tactile Internet," 2017 European Conference on Networks and Communications (EuCNC), 2017, pp. 1-5, doi: 10.1109/EuCNC.2017.7980645. 
[5] M. T. Vega, T. Mehmli, J. v. d. Hooft, T. Wauters and F. D. Turck, "Enabling Virtual Reality for the Tactile Internet: Hurdles and Opportunities," 2018 14th International Conference on Network and Service Management (CNSM), 2018, pp. 378-383.

[6] The Tactile Internet, ITU-T Technology Watch Report, August 2014, https://www.itu.int/dms_pub/itu-t/oth/23/01/T23010000230001PDFE.pdf, accessed online, November 2021

[7] P. Buttolo, R. Oboe, Blake Hannaford, Architectures for shared haptic virtual environments, Computers \& Graphics, Volume 21, Issue 4, 1997, pp. 421-429, ISSN 0097-8493,

[8] K. Salisbury, F. Conti and F. Barbagli, "Haptic rendering: introductory concepts," in IEEE Computer Graphics and Applications, vol. 24, no. 2, pp. 24-32, March-April 2004, doi: 10.1109/MCG.2004.1274058.

[9] Colum D. MacKinnon, Chapter 1 - Sensorimotor anatomy of gait, balance, and falls, Editor(s): Brian L. Day, Stephen R. Lord, Handbook of Clinical Neurology, Elsevier, Volume 159, 2018, Pages 3-26, ISSN 0072-9752, ISBN 9780444639165, https://doi.org/10.1016/B978-0-444-63916-5.00001-X.

[10] G. García-Valle, S. Arranz-Paraíso, I. Serrano-Pedraza and M. Ferre, "Estimation of Torso Vibrotactile Thresholds Using Eccentric Rotating Mass Motors," in IEEE Transactions on Haptics, vol. 14, no. 3, pp. 538-550, 1 July-Sept. 2021, doi: 10.1109/TOH.2020.3048290.

[11] G. G. Poyraz and Ö. Tamer, "Different Haptic Senses with Multiple Vibration Motors," 2019 11th International Conference on Electrical and Electronics Engineering (ELECO), 2019, pp. 870-874, doi: 10.23919/ELECO47770.2019.8990480.

[12] E. Hendler and J. D. Hardy, "Infrared and Microwave Effects on Skin Heating and Temperature Sensation," in IRE Transactions on Medical Electronics, vol. ME-7, no. 3, pp. 143-152, July 1960, doi: 10.1109/IRET-ME.1960.5008037

[13] N. Rendevski and D. Cassioli, "60 GHz UWB rake receivers in a realistic scenario for wireless home entertainment," 2015 IEEE International Conference on Communications (ICC), 2015, pp. 27442749, doi: 10.1109/ICC.2015.7248741.

[14] Rendevski N., Cassioli D. (2014) UWB and mmWave Communication Techniques and Systems for Healthcare. In: Yuce M. (eds) Ultra-Wideband and $60 \mathrm{GHz}$ Communications for Biomedical Applications. Springer, Boston, MA. https://doi.org/10.1007/978-1-4614-8896-5_1 